\title{
Some Generalized Fixed Point Results with Applications to Dynamic Programming
}

\author{
Hamed H. Al-Sulami, ${ }^{1}$ Nawab Hussain, ${ }^{1}$ and Jamshaid Ahmad ${ }^{2}{ }^{2}$ \\ ${ }^{1}$ Department of Mathematics, King Abdulaziz University, P.O. Box 80203, Jeddah 21589, Saudi Arabia \\ ${ }^{2}$ Department of Mathematics, University of Jeddah, P.O. Box 80327, Jeddah 21589, Saudi Arabia \\ Correspondence should be addressed to Jamshaid Ahmad; jamshaid_jasim@yahoo.com
}

Received 18 July 2020; Revised 25 August 2020; Accepted 14 September 2020; Published 26 September 2020

Academic Editor: Gestur lafsson

Copyright (c) 2020 Hamed H. Al-Sulami et al. This is an open access article distributed under the Creative Commons Attribution License, which permits unrestricted use, distribution, and reproduction in any medium, provided the original work is properly cited.

The aim of this paper is to introduce some generalized contractions and prove certain new fixed point results for self-mappings satisfying these contractions in the setting of $\mathscr{F}$-metric space. As an application of our results, we investigate the problem of dynamic programming related to the multistage process which formulates the problems of computer programming and mathematical optimization. We also provide an example to support the validity of our main results.

\section{Introduction}

Because of utility and applications of metric fixed point theory in mathematics and related fields like social sciences, physical sciences, computer sciences, and engineering have grown in many directions. More general theorems have been introduced along with the provision of many useful tools to solve problems arising in several diverse areas of research.

Recently, Jlei and Samet [1] initiated a generalized metric space named as $\mathscr{F}$-metric space and showed a generalization of the Banach contraction principle. Meanwhile, researchers have picked keen interests in extending results in this generalized metric space; see for instance, [2-5]. In this paper, we define some generalized contractions and establish some results in the context of $\mathscr{F}$-metric spaces.

\section{Preliminaries}

Here, we record some requisites definitions and results for the purpose of the next sections.

Definition 1 (see [1]). Let $\mathscr{F}$ be the class of functions $f:(0$, $+\infty) \longrightarrow \mathbb{R}$ satisfying these assertions:

$$
\begin{aligned}
& \left(\mathscr{F}_{1}\right) 0<s<\iota=\Longrightarrow f(s) \leq f(\iota) \\
& \left(\mathscr{F}_{2}\right) \forall\left\{\iota_{n}\right\} \subseteq R^{+}, \lim _{n \rightarrow \infty} \iota_{n}=0 \Leftrightarrow \lim _{n \rightarrow \infty} f\left(\iota_{n}\right)=-\infty
\end{aligned}
$$

Example 2. If $f:(0,+\infty) \longrightarrow \mathbb{R}$ are defined as.

(1) $f(\mathrm{Q})=\ln (\mathrm{Q})$

(2) $f(\mathrm{Q})=\mathrm{Q}+\ln (\mathrm{Q})$

(3) $f(\mathrm{e})=-1 / \sqrt{\mathrm{\varrho}}$

(4) $f(\mathrm{Q})=\ln \left(\mathrm{Q}^{2}+\mathrm{Q}\right)$

for $\mathrm{\varrho}>0$, then $f \in \mathscr{F}$.

Definition 3 (see [1]). Let $\mathscr{M} \neq \varnothing$, and $d_{\mathscr{F}}: \mathscr{M} \times \mathscr{M} \longrightarrow 0$, $+\infty)$. Assume that $\exists(f, \tau) \in \mathscr{F} \times[0,+\infty)$ such that

$\left(\mathrm{D}_{1}\right)$ for all $\zeta, \xi \in \mathscr{M}, d_{\mathscr{F}}(\zeta, \xi)=0 \Leftrightarrow \zeta=\xi$

$\left(\mathrm{D}_{2}\right) d_{\mathscr{F}}(\zeta, \xi)=d_{\mathscr{F}}(\xi, \zeta)$, for all $(\zeta, \xi) \in \mathscr{M} \times \mathscr{M}$

$\left(\mathrm{D}_{3}\right)$ for every $(\zeta, \xi) \in \mathscr{M} \times \mathscr{M}$, for every $N \in \mathbb{N}, N \geq 2$, and $\left(\mathfrak{w}_{i}\right)_{i=1}^{N} \subset \mathscr{M}$, with $\left(\mathfrak{w}_{1}, \mathfrak{w}_{N}\right)=(\zeta, \xi)$, we have

$$
d_{\mathscr{F}}(\zeta, \xi)>0 \Longrightarrow f\left(d_{\mathscr{F}}(\zeta, \xi)\right) \leq f\left(\sum_{i=1}^{N-1} d_{\mathscr{F}}\left(\zeta_{i}, \zeta_{i+1}\right)\right)+\tau
$$

Then, $\left(\mathscr{M}, d_{\mathscr{F}}\right)$ is called an $\mathscr{F}$-metric space. 
Example 4 (see [1]). Let $d_{\mathscr{F}}: \mathbb{R} \times \mathbb{R} \longrightarrow \mathbb{R}^{+}$be defined by

$$
d_{\mathscr{F}}(\zeta, \xi)=\left\{\begin{array}{l}
(\zeta-\xi)^{2} \text { if }(\zeta, \xi) \in[0,3] \times[0,3] \\
|\zeta-\xi| \text { if }(\zeta, \xi) \in[0,3] \times[0,3]
\end{array}\right.
$$

with $f(\varrho)=\ln (\varrho)$ and $\tau=\ln (3)$. Then, $\left(\mathbb{R}, d_{\mathscr{F}}\right)$ is an $\mathscr{F}$-metric space.

Theorem 5. [1].

Let $\left(\mathscr{M}, d_{\mathscr{F}}\right)$ be an $\mathscr{F}$-metric space and $\mathscr{J}: \mathscr{M} \longrightarrow \mathscr{M}$. Assume that these conditions are satisfied:

(i) $\left(\mathscr{M}, d_{\mathscr{F}}\right)$ is $\mathscr{F}$-complete

(ii) $\exists 0<\lambda<1$ such that

$$
d_{\mathscr{F}}(\mathscr{J}(\zeta), \mathscr{J}(\xi)) \leq \lambda d_{\mathscr{F}}(\zeta, \xi)
$$

Then, $\exists \zeta^{*} \in \mathscr{M}$ such that $\mathscr{J} \zeta^{*}=\zeta^{*}$. Furthermore, for any $\zeta_{0} \in \mathscr{M},\left\{\zeta_{n}\right\} \subset \mathscr{M}$ defined by

$$
\zeta_{n+1}=\mathscr{J}\left(\zeta_{n}\right), n \in \mathbb{N}
$$

is $\mathscr{F}$-convergent to $\zeta^{*}$.

Afterwards, many researchers [2-9] worked in this space.

In this article, we give the notions of twisted $(\alpha, \beta)$ -admissible and twisted $(\alpha, \beta)-\varphi$-rational contractions in the setting of $\mathscr{F}$-metric spaces and prove some new theorems.

\section{Main Results}

In 2012, Samet et al. [10] introduced the concepts of $\alpha$-admissibility mappings and $\alpha$ - $\varphi$-contraction in complete metric spaces.

Definition 6 (see [10]). Let $\mathscr{J}: \mathscr{M} \longrightarrow \mathscr{M}$ and $\alpha: \mathscr{M} \times \mathscr{M}$ $\longrightarrow 0,+\infty)$. Then, $\mathscr{J}$ is said to be $\alpha$-admissible if $\zeta, \xi \in \mathscr{M}$,

$$
\alpha(\zeta, \xi) \geq 1 \Longrightarrow \alpha(\mathscr{J} \zeta, \mathscr{J} \xi) \geq 1
$$

According to Samet et al. [10], $\Psi$ represents the class of all nondecreasing functions $\varphi:[0,+\infty) \longrightarrow 0,+\infty)$ such that $\sum_{n=1}^{\infty} \varphi^{n}(\iota)<+\infty$ for all $\iota>0$, where $\varphi^{n}$ is the $n$th iterate of $\varphi$.

Lemma 7 (see [10]). If $\varphi \in \Psi$, then

$$
\begin{aligned}
& \text { (i) }\left(\varphi^{n}(\iota)\right)_{n \in \mathbb{N}} \longrightarrow 0 \text { as } n \longrightarrow \infty, \forall \iota \in(0,+\infty) \\
& \text { (ii) } \varphi(\iota)<\iota \text { for all } \iota>0 \\
& \text { (iii) } \varphi(\iota)=0 \text { iff } \iota=0
\end{aligned}
$$

Now, we give the concept of twisted $(\alpha, \beta)$-admissible in the $\mathscr{F}$-metric space as follows.

Definition 8. Let $\left(\mathscr{M}, d_{\mathscr{F}}\right)$ be an $\mathscr{F}$-metric space, $\mathscr{J}: \mathscr{M}$ $\longrightarrow \mathscr{M}$ and $\alpha, \beta: \mathscr{M} \times \mathscr{M} \longrightarrow 0,+\infty)$. Then, $\mathscr{J}$ is said to be twisted $(\alpha, \beta)$-admissible if

$$
\left\{\begin{array} { l } 
{ \alpha ( \zeta , \xi ) \geq 1 } \\
{ \beta ( \zeta , \xi ) \geq 1 }
\end{array} \Longrightarrow \left\{\begin{array}{l}
\alpha(\mathscr{J} \zeta, \mathscr{J} \xi) \geq 1 \\
\beta(\mathscr{J} \zeta, \mathscr{J} \xi) \geq 1
\end{array}\right.\right.
$$

for $\zeta, \xi \in \mathscr{M}$.

Now, we state our main result.

Theorem 9. Let $\left(\mathscr{M}, d_{\mathscr{F}}\right)$ be an $\mathscr{F}$-metric space and $\mathscr{J}: \mathscr{M}$ $\longrightarrow \mathscr{M}$ be twisted $(\alpha, \beta)$-admissible. Suppose that the following assertions are satisfied:

(a) $\left(\mathscr{M}, d_{\mathscr{F}}\right)$ is $\mathscr{F}$-complete

(b) there exists $\zeta_{0} \in \mathscr{M}$ such that $\alpha\left(\zeta_{0}, \mathscr{J}\left(\zeta_{0}\right)\right) \geq 1$ and $\beta$ $\left(\zeta_{0}, \mathscr{J}\left(\zeta_{0}\right)\right) \geq 1$

(c) $\mathcal{J}$ is continuous. If any one of these assertions hold:

$$
\alpha(\zeta, \xi) \beta(\zeta, \xi) d_{\mathscr{F}}(\mathscr{J}(\zeta), \mathscr{J}(\xi)) \leq \varphi(\mathscr{R}(\zeta, \xi))
$$

(i) $\exists 0<l \leq 1$ such that

$$
(\alpha(\zeta, \xi) \beta(\zeta, \xi)+l)^{d_{\mathscr{F}}(\mathscr{F}(\zeta), \mathscr{F}(\xi))} \leq(1+l)^{\varphi(\mathscr{R}(\zeta, \xi))}
$$

(ii) $\exists l \geq 1$ such that

$$
\left(d_{\mathscr{F}}(\mathscr{J}(\zeta), \mathscr{J}(\xi))+l\right)^{\alpha(\zeta, \xi) \beta(\zeta, \xi)} \leq \varphi(\mathscr{R}(\zeta, \xi))+l
$$

where

$$
\mathscr{R}(\zeta, \xi)=\max \left\{d_{\mathscr{F}}(\zeta, \xi), \frac{d_{\mathscr{F}}(\zeta, \mathscr{J}(\zeta)) d_{\mathscr{F}}(\xi, \mathscr{J}(\xi))}{1+d_{\mathscr{F}}(\zeta, \xi)}\right\}
$$

for all $\zeta, \xi \in \mathscr{M}$, then $\exists \zeta^{*} \in \mathscr{M}$ such that $\mathscr{J} \zeta^{*}=\zeta^{*}$.

Proof. Let $\zeta_{0} \in \mathscr{M}$ such that $\alpha\left(\zeta_{0}, \mathscr{J}\left(\zeta_{0}\right)\right) \geq 1$ and $\beta\left(\zeta_{0}, \mathscr{J}\right.$ $\left.\left(\zeta_{0}\right)\right) \geq 1$. Generate $\left\{\zeta_{n}\right\}$ in $\mathscr{M}$ by $\zeta_{n+1}=\mathscr{J}\left(\zeta_{n}\right), \forall n \in \mathbb{N}$. If $\zeta_{n+1}=\zeta_{n}$ for some $n \in \mathbb{N}$, then $\zeta^{*}=\zeta_{n}$ is a fixed point of $\mathscr{J}$. So we suppose that $\zeta_{n+1} \neq, \forall n \in \mathbb{N}$. Then as $\mathscr{J}$ is twisted $(\alpha, \beta)$-admissible, we get $\alpha\left(\zeta_{0}, \zeta_{1}\right)=\alpha\left(\zeta_{0}, \mathscr{J}\left(\zeta_{0}\right)\right)$ $\geq 1$ implies $\alpha\left(\zeta_{1}, \zeta_{2}\right)=\alpha\left(\mathscr{J}\left(\zeta_{0}\right), \mathscr{J}\left(\zeta_{1}\right)\right) \geq 1$ and $\beta\left(\zeta_{1}, \zeta_{2}\right)$ $=\beta\left(\mathscr{J}\left(\zeta_{0}\right), \mathscr{J}\left(\zeta_{1}\right)\right) \geq 1$. By induction, we get $\alpha\left(\zeta_{n}, \zeta_{n+1}\right)$ $\geq 1$ and $\beta\left(\zeta_{n}, \zeta_{n+1}\right) \geq 1$ for all $n \in \mathbb{N}$. Suppose the inequality (7) holds. So with $\zeta=\zeta_{n-1}$ and $\xi=\zeta_{n}$, we have

$$
\begin{aligned}
d_{\mathscr{F}}\left(\zeta_{n}, \zeta_{n+1}\right)= & d_{\mathscr{F}}\left(\mathscr{J}\left(\zeta_{n-1}\right), \mathscr{J}\left(\zeta_{n}\right)\right) \\
& \leq \alpha\left(\zeta_{n-1}, \zeta_{n}\right) \beta\left(\zeta_{n-1}, \zeta_{n}\right) d_{\mathscr{F}}\left(\mathscr{J}\left(\zeta_{n-1}\right), \mathscr{J}\left(\zeta_{n}\right)\right) \\
& \leq \varphi\left(\mathscr{R}\left(\zeta_{n-1}, \zeta_{n}\right)\right)
\end{aligned}
$$


where

$$
\begin{aligned}
\mathscr{R}\left(\zeta_{n-1}, \zeta_{n}\right) & =\max \left\{d_{\mathscr{F}}\left(\zeta_{n-1}, \zeta_{n}\right), \frac{d_{\mathscr{F}}\left(\zeta_{n-1}, \mathscr{J}\left(\zeta_{n-1}\right)\right) d_{\mathscr{F}}\left(\zeta_{n}, \mathscr{J}\left(\zeta_{n}\right)\right)}{1+d_{\mathscr{F}}\left(\zeta_{n-1}, \zeta_{n}\right)}\right\} \\
& =\max \left\{d_{\mathscr{F}}\left(\zeta_{n-1}, \zeta_{n}\right), \frac{d_{\mathscr{F}}\left(\zeta_{n-1}, \zeta_{n}\right) d_{\mathscr{F}}\left(\zeta_{n}, \zeta_{n+1}\right)}{1+d_{\mathscr{F}}\left(\zeta_{n-1}, \zeta_{n}\right)}\right\}
\end{aligned}
$$

If $\max \left\{d_{\mathscr{F}}\left(\zeta_{n-1}, \zeta_{n}\right),\left(d_{\mathscr{F}}\left(\zeta_{n-1}, \zeta_{n}\right) d_{\mathscr{F}}\left(\zeta_{n}, \zeta_{n+1}\right)\right) /\left(1+d_{\mathscr{F}}\right.\right.$ $\left.\left.\left(\zeta_{n-1}, \zeta_{n}\right)\right)\right\}=\left(d_{\mathscr{F}}\left(\zeta_{n-1}, \zeta_{n}\right) d_{\mathscr{F}}\left(\zeta_{n}, \zeta_{n+1}\right)\right) /\left(1+d_{\mathscr{F}}\left(\zeta_{n-1}, \zeta_{n}\right)\right)$, then from (11), we obtain

$$
\begin{aligned}
d_{\mathscr{F}}\left(\zeta_{n}, \zeta_{n+1}\right) & \leq \varphi\left(\frac{d_{\mathscr{F}}\left(\zeta_{n-1}, \zeta_{n}\right) d_{\mathscr{F}}\left(\zeta_{n}, \zeta_{n+1}\right)}{1+d_{\mathscr{F}}\left(\zeta_{n-1}, \zeta_{n}\right)}\right) \\
& <\frac{d_{\mathscr{F}}\left(\zeta_{n-1}, \zeta_{n}\right) d_{\mathscr{F}}\left(\zeta_{n}, \zeta_{n+1}\right)}{1+d_{\mathscr{F}}\left(\zeta_{n-1}, \zeta_{n}\right)} \leq d_{\mathscr{F}}\left(\zeta_{n}, \zeta_{n+1}\right)
\end{aligned}
$$

a contradiction. Hence, $\max \left\{d_{\mathscr{F}}\left(\zeta_{n-1}, \zeta_{n}\right),\left(d_{\mathscr{F}}\left(\zeta_{n-1}, \zeta_{n}\right)\right.\right.$ $\left.\left.d_{\mathscr{F}}\left(\zeta_{n}, \zeta_{n+1}\right)\right) /\left(1+d_{\mathscr{F}}\left(\zeta_{n-1}, \zeta_{n}\right)\right)\right\}=d_{\mathscr{F}}\left(\zeta_{n-1}, \zeta_{n}\right)$; therefore, (11) becomes

$$
d_{\mathscr{F}}\left(\zeta_{n}, \zeta_{n+1}\right) \leq \varphi\left(d_{\mathscr{F}}\left(\zeta_{n-1}, \zeta_{n}\right)\right)
$$

Consequently, we get

$$
d_{\mathscr{F}}\left(\zeta_{n}, \zeta_{n+1}\right) \leq \varphi^{n}\left(d_{\mathscr{F}}\left(\zeta_{0}, \zeta_{1}\right)\right)
$$

$\forall n \in \mathbb{N}$.

Assume inequality (8) holds and $\exists 0<l \leq 1$ such that

$$
\begin{aligned}
(1+l)^{d_{\mathscr{F}}\left(\zeta_{n}, \zeta_{n+1}\right)} & \leq\left(\alpha\left(\zeta_{n-1}, \zeta_{n}\right) \beta\left(\zeta_{n-1}, \zeta_{n}\right)+l\right)^{d_{\mathscr{F}}\left(\mathscr{F}\left(\zeta_{n-1}\right), \mathscr{F}\left(\zeta_{n}\right)\right)} \\
& \leq(1+l)^{\varphi\left(\mathscr{R}\left(\zeta_{n-1}, \zeta_{n}\right)\right)}
\end{aligned}
$$

which implies that

$$
d_{\mathscr{F}}\left(\zeta_{n}, \zeta_{n+1}\right) \leq \varphi\left(\mathscr{R}\left(\zeta_{n-1}, \zeta_{n}\right)\right)
$$

where

$$
\begin{aligned}
\mathscr{R}\left(\zeta_{n-1}, \zeta_{n}\right) & =\max \left\{d_{\mathscr{F}}\left(\zeta_{n-1}, \zeta_{n}\right), \frac{d_{\mathscr{F}}\left(\zeta_{n-1}, \mathscr{J}\left(\zeta_{n-1}\right)\right) d_{\mathscr{F}}\left(\zeta_{n}, \mathscr{J}\left(\zeta_{n}\right)\right)}{1+d_{\mathscr{F}}\left(\zeta_{n-1}, \zeta_{n}\right)}\right\} \\
& =\max \left\{d_{\mathscr{F}}\left(\zeta_{n-1}, \zeta_{n}\right), \frac{d_{\mathscr{F}}\left(\zeta_{n-1}, \zeta_{n}\right) d_{\mathscr{F}}\left(\zeta_{n}, \zeta_{n+1}\right)}{1+d_{\mathscr{F}}\left(\zeta_{n-1}, \zeta_{n}\right)}\right\}
\end{aligned}
$$

If $\max \left\{d_{\mathscr{F}}\left(\zeta_{n-1}, \zeta_{n}\right),\left(d_{\mathscr{F}}\left(\zeta_{n-1}, \zeta_{n}\right) d_{\mathscr{F}}\left(\zeta_{n}, \zeta_{n+1}\right)\right) /\left(1+d_{\mathscr{F}}\right.\right.$ $\left.\left.\left(\zeta_{n-1}, \zeta_{n}\right)\right)\right\}=\left(d_{\mathscr{F}}\left(\zeta_{n-1}, \zeta_{n}\right) d_{\mathscr{F}}\left(\zeta_{n}, \zeta_{n+1}\right)\right) /\left(1+d_{\mathscr{F}}\left(\zeta_{n-1}, \zeta_{n}\right)\right)$, then from (17), we obtain

$$
\begin{aligned}
d_{\mathscr{F}}\left(\zeta_{n}, \zeta_{n+1}\right) & \leq \varphi\left(\frac{d_{\mathscr{F}}\left(\zeta_{n-1}, \zeta_{n}\right) d_{\mathscr{F}}\left(\zeta_{n}, \zeta_{n+1}\right)}{1+d_{\mathscr{F}}\left(\zeta_{n-1}, \zeta_{n}\right)}\right) \\
& <\frac{d_{\mathscr{F}}\left(\zeta_{n-1}, \zeta_{n}\right) d_{\mathscr{F}}\left(\zeta_{n}, \zeta_{n+1}\right)}{1+d_{\mathscr{F}}\left(\zeta_{n-1}, \zeta_{n}\right)} \leq d_{\mathscr{F}}\left(\zeta_{n}, \zeta_{n+1}\right),
\end{aligned}
$$

a contradiction. Thus, $\max \left\{d_{\mathscr{F}}\left(\zeta_{n-1}, \zeta_{n}\right),\left(d_{\mathscr{F}}\left(\zeta_{n-1}, \zeta_{n}\right) d_{\mathscr{F}}\right.\right.$ $\left.\left.\left(\zeta_{n}, \zeta_{n+1}\right)\right) /\left(1+d_{\mathscr{F}}\left(\zeta_{n-1}, \zeta_{n}\right)\right)\right\}=d_{\mathscr{F}}\left(\zeta_{n-1}, \zeta_{n}\right)$; therefore, (17) becomes

$$
d_{\mathscr{F}}\left(\zeta_{n}, \zeta_{n+1}\right) \leq \varphi\left(d_{\mathscr{F}}\left(\zeta_{n-1}, \zeta_{n}\right)\right)
$$

Consequently, we get

$$
d_{\mathscr{F}}\left(\zeta_{n}, \zeta_{n+1}\right) \leq \varphi^{n}\left(d_{\mathscr{F}}\left(\zeta_{0}, \zeta_{1}\right)\right)
$$

Assume inequality (9) holds and $\exists l \leq 1$ such that

$$
\begin{aligned}
\left(d_{\mathscr{F}}\left(\zeta_{n}, \zeta_{n+1}\right)+l\right) & \leq\left(d_{\mathscr{F}}\left(\mathscr{J}\left(\zeta_{n-1}\right), \mathscr{J}\left(\zeta_{n}\right)\right)+l\right)^{\alpha\left(\zeta_{n-1}, \zeta_{n}\right) \beta\left(\zeta_{n-1}, \zeta_{n}\right)} \\
& \leq \varphi\left(\mathscr{R}\left(\zeta_{n-1}, \zeta_{n}\right)\right)+l
\end{aligned}
$$

which implies that

$$
d_{\mathscr{F}}\left(\zeta_{n}, \zeta_{n+1}\right) \leq \varphi\left(\mathscr{R}\left(\zeta_{n-1}, \zeta_{n}\right)\right)
$$

where

$$
\begin{aligned}
\mathscr{R}\left(\zeta_{n-1}, \zeta_{n}\right) & =\max \left\{d_{\mathscr{F}}\left(\zeta_{n-1}, \zeta_{n}\right), \frac{d_{\mathscr{F}}\left(\zeta_{n-1}, \mathscr{J}\left(\zeta_{n-1}\right)\right) d_{\mathscr{F}}\left(\zeta_{n}, \mathscr{J}\left(\zeta_{n}\right)\right)}{1+d_{\mathscr{F}}\left(\zeta_{n-1}, \zeta_{n}\right)}\right\} \\
& =\max \left\{d_{\mathscr{F}}\left(\zeta_{n-1}, \zeta_{n}\right), \frac{d_{\mathscr{F}}\left(\zeta_{n-1}, \zeta_{n}\right) d_{\mathscr{F}}\left(\zeta_{n}, \zeta_{n+1}\right)}{1+d_{\mathscr{F}}\left(\zeta_{n-1}, \zeta_{n}\right)}\right\}
\end{aligned}
$$

If $\max \left\{d_{\mathscr{F}}\left(\zeta_{n-1}, \zeta_{n}\right),\left(d_{\mathscr{F}}\left(\zeta_{n-1}, \zeta_{n}\right) d_{\mathscr{F}}\left(\zeta_{n}, \zeta_{n+1}\right)\right) /\left(1+d_{\mathscr{F}}\right.\right.$ $\left.\left.\left(\zeta_{n-1}, \zeta_{n}\right)\right)\right\}=\left(d_{\mathscr{F}}\left(\zeta_{n-1}, \zeta_{n}\right) d_{\mathscr{F}}\left(\zeta_{n}, \zeta_{n+1}\right)\right) /\left(1+d_{\mathscr{F}}\left(\zeta_{n-1}, \zeta_{n}\right)\right)$, then from (23), we obtain

$$
\begin{aligned}
d_{\mathscr{F}}\left(\zeta_{n}, \zeta_{n+1}\right) & \leq \varphi\left(\frac{d_{\mathscr{F}}\left(\zeta_{n-1}, \zeta_{n}\right) d_{\mathscr{F}}\left(\zeta_{n}, \zeta_{n+1}\right)}{1+d_{\mathscr{F}}\left(\zeta_{n-1}, \zeta_{n}\right)}\right) \\
& <\frac{d_{\mathscr{F}}\left(\zeta_{n-1}, \zeta_{n}\right) d_{\mathscr{F}}\left(\zeta_{n}, \zeta_{n+1}\right)}{1+d_{\mathscr{F}}\left(\zeta_{n-1}, \zeta_{n}\right)} \leq d_{\mathscr{F}}\left(\zeta_{n}, \zeta_{n+1}\right),
\end{aligned}
$$

a contradiction. Thus, $\max \left\{d_{\mathscr{F}}\left(\zeta_{n-1}, \zeta_{n}\right),\left(d_{\mathscr{F}}\left(\zeta_{n-1}, \zeta_{n}\right)\right.\right.$ $\left.\left.d_{\mathscr{F}}\left(\zeta_{n}, \zeta_{n+1}\right)\right) /\left(1+d_{\mathscr{F}}\left(\zeta_{n-1}, \zeta_{n}\right)\right)\right\}=d_{\mathscr{F}}\left(\zeta_{n-1}, \zeta_{n}\right)$; therefore, (23) becomes

$$
d_{\mathscr{F}}\left(\zeta_{n}, \zeta_{n+1}\right) \leq \varphi\left(d_{\mathscr{F}}\left(\zeta_{n-1}, \zeta_{n}\right)\right)
$$

Consequently, we get

$$
d_{\mathscr{F}}\left(\zeta_{n}, \zeta_{n+1}\right) \leq \varphi^{n}\left(d_{\mathscr{F}}\left(\zeta_{0}, \zeta_{1}\right)\right) \text {, }
$$


Let $(f, \tau) \in \mathscr{F} \times 0,+\infty)$ be such that $\left(\mathrm{D}_{3}\right)$ is satisfied. Let $\varepsilon>0$ be fixed. By $\left(\mathscr{F}_{2}\right), \exists \delta>0$ such that

$$
0<\iota<\delta \Longrightarrow f(\iota)<f(\delta)-\tau \text {. }
$$

Let $n(\varepsilon) \in \mathbb{N}$ such that $0<\sum_{n \geq n(\varepsilon)} \varphi^{n}\left(d_{\mathscr{F}}\left(\zeta_{0}, \zeta_{1}\right)\right)<\delta$. Hence, by $(27),\left(\mathscr{F}_{1}\right)$, and $\left(\mathscr{F}_{2}\right)$, we have

$$
\begin{aligned}
f\left(\sum_{i=n}^{m-1} d_{\mathscr{F}}\left(\zeta_{i}, \zeta_{i+1}\right)\right) & \leq f\left(\sum_{i=n}^{m-1} \varphi^{i}\left(d_{\mathscr{F}}\left(\zeta_{0}, \zeta_{1}\right)\right)\right) \\
& \leq f\left(\sum_{n \geq n(\varepsilon)} \varphi^{n}\left(d_{\mathscr{F}}\left(\zeta_{0}, \zeta_{1}\right)\right)\right)<f(\varepsilon)-\tau,
\end{aligned}
$$

for $m>n \geq n(\varepsilon)$. Using $\left(\mathrm{D}_{3}\right)$ and (29), we get $d_{\mathscr{F}}\left(\zeta_{n}, \zeta_{m}\right)>0$, $m>n \geq n(\varepsilon)$ implies

$$
f\left(d_{\mathscr{F}}\left(\zeta_{n}, \zeta_{m}\right)\right) \leq f\left(\sum_{i=n}^{m-1} d_{\mathscr{F}}\left(\zeta_{i}, \zeta_{i+1}\right)\right)+\tau<f(\varepsilon)
$$

which implies by $\left(\mathscr{F}_{1}\right)$ that $d_{\mathscr{F}}\left(\zeta_{n}, \zeta_{m}\right)<\varepsilon, m>n \geq n(\varepsilon)$. This shows that $\left\{\zeta_{n}\right\}$ is $\mathscr{F}$-Cauchy. As $\left(\mathscr{M}, d_{\mathscr{F}}\right)$ is $\mathscr{F}$-complete, $\exists$ $\zeta^{*} \in \mathscr{M}$ such that $\left\{\zeta_{n}\right\}$ is $\mathscr{F}$-convergent to $\zeta^{*}$. As $\mathscr{J}$ is continuous, so we have $\mathscr{J}\left(\zeta^{*}\right)=\lim _{n \rightarrow \infty} \mathscr{J}\left(\zeta_{n}\right) \ll \lim _{n \rightarrow \infty} \zeta_{n+1}=\zeta^{*}$. Thus, $\exists \zeta^{*} \in \mathscr{M}$ such that $\mathscr{J} \zeta^{*}=\zeta^{*}$.

In the next result, we omit the continuity of $\mathscr{J}$ and use an adjunctive condition on $\mathscr{M}$.

Theorem 10. Let $\left(\mathscr{M}, d_{\mathscr{F}}\right)$ be an $\mathscr{F}$-metric space and $\mathscr{J}: \mathscr{M} \longrightarrow \mathscr{M}$ be twisted $(\alpha, \beta)$-admissible. Suppose that the following assertions are satisfied:

(a) $\left(\mathscr{M}, d_{\mathscr{F}}\right)$ is $\mathscr{F}$-complete

(b) $\exists \zeta_{0} \in \mathscr{M}$ such that $\alpha\left(\zeta_{0}, \mathscr{J}\left(\zeta_{0}\right)\right) \geq 1$ and $\beta\left(\zeta_{0}\right.$, $\left.\mathscr{J}\left(\zeta_{0}\right)\right) \geq 1$

(c) If $\left\{\zeta_{n}\right\}$ is a sequence in $\mathscr{M}$ such that $\alpha\left(\zeta_{n}, \zeta_{n+1}\right) \geq 1$ and $\beta\left(\zeta_{n}, \zeta_{n+1}\right) \geq 1, \forall n$ and $\zeta_{n} \longrightarrow \zeta^{*} \in \mathscr{M}$ as $n \longrightarrow$ $\infty$, then $\alpha\left(\zeta_{n}, \zeta^{*}\right) \geq 1$ and $\beta\left(\zeta_{n}, \zeta^{*}\right) \geq 1, \forall n \in \mathbb{N}$

If any one of these assertions hold:

$$
\alpha(\zeta, \xi) \beta(\zeta, \xi) d_{\mathscr{F}}(\mathscr{J}(\zeta), \mathscr{J}(\xi)) \leq \varphi(\mathscr{R}(\zeta, \xi)),
$$

(i) $\exists 0<l \leq 1$ such that

$$
(\alpha(\zeta, \xi) \beta(\zeta, \xi)+l)^{d_{\mathscr{F}}(\mathscr{F}(\zeta), \mathscr{F}(\xi))} \leq(1+l)^{\varphi(\mathscr{R}(\zeta, \xi))}
$$

(ii) $\exists l \geq 1$ such that

$$
\left(d_{\mathscr{F}}(\mathscr{J}(\zeta), \mathscr{J}(\xi))+l\right)^{\alpha(\zeta, \xi) \beta(\zeta, \xi)} \leq \varphi(\mathscr{R}(\zeta, \xi))+l
$$

where

$$
\mathscr{R}(\zeta, \xi)=\max \left\{d_{\mathscr{F}}(\zeta, \xi), \frac{d_{\mathscr{F}}(\zeta, \mathscr{J}(\zeta)) d_{\mathscr{F}}(\xi, \mathscr{J}(\xi))}{1+d_{\mathscr{F}}(\zeta, \xi)}\right\},
$$

for all $\zeta, \xi \in \mathscr{M}$, then $\exists \zeta^{*} \in \mathscr{M}$ such that $\mathscr{J} \zeta^{*}=\zeta^{*}$.

Proof. $>$ Let $\zeta_{0} \in \mathscr{M}$ such that $\alpha\left(\zeta_{0}, \mathscr{J}\left(\zeta_{0}\right)\right) \geq 1$ and $\beta\left(\zeta_{0}, \mathscr{J}(\right.$ $\left.\left.\zeta_{0}\right)\right) \geq 1$. Proceeding as in the proof of Theorem 9, we have $\zeta^{*} \in \mathscr{M}$ such that $\left\{\zeta_{n}\right\}$ is $\mathscr{F}$-convergent to $\zeta^{*}$, i.e.,

$$
\lim _{n \rightarrow \infty} d_{\mathscr{F}}\left(\zeta_{n}, \zeta^{*}\right)=0
$$

Suppose that $d_{\mathscr{F}}\left(\mathscr{J}\left(\zeta^{*}\right), \zeta^{*}\right)>0$ and inequality (31) holds. By $\left(\mathscr{F}_{1}\right)$ and $\left(\mathrm{D}_{3}\right)$, we have

$$
\begin{aligned}
& d_{\mathscr{F}}\left(\mathscr{J}\left(\zeta^{*}\right), \zeta_{n+1}\right)=d_{\mathscr{F}}\left(\mathscr{J}\left(\zeta^{*}\right), \mathscr{J}\left(\zeta_{n}\right)\right) \\
& \quad \leq \alpha\left(\zeta^{*}, \zeta_{n}\right) \beta\left(\zeta^{*}, \zeta_{n}\right) d_{\mathscr{F}}\left(\mathscr{J}\left(\zeta^{*}\right), \mathscr{J}\left(\zeta_{n}\right)\right) \\
& \quad \leq \varphi\left(\max \left\{d_{\mathscr{F}}\left(\zeta^{*}, \zeta_{n}\right), \frac{d_{\mathscr{F}}\left(\zeta^{*}, \mathscr{J}\left(\zeta^{*}\right)\right) d_{\mathscr{F}}\left(\zeta_{n}, \mathscr{J}\left(\zeta_{n}\right)\right)}{1+d_{\mathscr{F}}\left(\zeta^{*}, \zeta_{n}\right)}\right\}\right) .
\end{aligned}
$$

Similarly, if inequality (32) holds. So $\exists 0<l \leq 1$ such that

$$
\begin{aligned}
(1+l)^{d_{\mathscr{F}}\left(\mathscr{F}\left(\zeta^{*}\right), \zeta_{n+1}\right)} & =(1+l)^{d_{\mathscr{F}}\left(\mathscr{F}\left(\zeta^{*}\right), \mathscr{F}\left(\zeta_{n}\right)\right)} \\
& \leq\left(\alpha\left(\zeta^{*}, \zeta_{n}\right) \beta\left(\zeta^{*}, \zeta_{n}\right)+l\right)^{d_{\mathscr{F}}\left(\mathscr{F}\left(\zeta^{*}\right), \mathscr{F}\left(\zeta_{n}\right)\right)} \\
& \leq(1+l)^{\varphi\left(\mathscr{R}\left(\zeta^{*}, \zeta_{n}\right)\right)},
\end{aligned}
$$

which implies that

$$
d_{\mathscr{F}}\left(\mathscr{J}\left(\zeta^{*}\right), \zeta_{n+1}\right) \leq \varphi\left(\mathscr{R}\left(\zeta^{*}, \zeta_{n}\right)\right)
$$

that is

$d_{\mathscr{F}}\left(\mathscr{J}\left(\zeta^{*}\right), \zeta_{n+1}\right) \leq \varphi\left(\max \left\{d_{\mathscr{F}}\left(\zeta^{*}, \zeta_{n}\right), \frac{d_{\mathscr{F}}\left(\zeta^{*}, \mathscr{J}\left(\zeta^{*}\right)\right) d_{\mathscr{F}}\left(\zeta_{n}, \mathscr{J}\left(\zeta_{n}\right)\right)}{1+d_{\mathscr{F}}\left(\zeta^{*}, \zeta_{n}\right)}\right\}\right)$.

Also if inequality (33) holds, then $\exists l \geq 1$ such that

$$
\begin{aligned}
\left(d_{\mathscr{F}}\left(\mathscr{J}\left(\zeta^{*}\right), \zeta_{n+1}\right)+l\right) & \leq\left(d_{\mathscr{F}}\left(\mathscr{J}\left(\zeta^{*}\right), \mathscr{J}\left(\zeta_{n}\right)\right)+l\right)^{\alpha\left(\zeta^{*}, \zeta_{n}\right) \beta\left(\zeta^{*}, \zeta_{n}\right)} \\
& \leq \varphi\left(\mathscr{R}\left(\zeta^{*}, \zeta_{n}\right)\right)+l d_{\mathscr{F}}\left(\mathscr{J}\left(\zeta^{*}\right), \zeta_{n+1}\right) \\
& \leq \varphi\left(\mathscr{R}\left(\zeta^{*}, \zeta_{n}\right)\right)
\end{aligned}
$$

that is

$$
d_{\mathscr{F}}\left(\mathscr{J}\left(\zeta^{*}\right), \zeta_{n+1}\right) \leq \varphi\left(\max \left\{d_{\mathscr{F}}\left(\zeta^{*}, \zeta_{n}\right), \frac{d_{\mathscr{F}}\left(\zeta^{*}, \mathscr{J}\left(\zeta^{*}\right)\right) d_{\mathscr{F}}\left(\zeta_{n}, \mathscr{J}\left(\zeta_{n}\right)\right)}{1+d_{\mathscr{F}}\left(\zeta^{*}, \zeta_{n}\right)}\right\}\right) .
$$


Thus, for all cases, by $\left(\mathscr{F}_{1}\right)$ and $\left(\mathrm{D}_{3}\right)$, we have

$$
\begin{aligned}
f\left(d_{\mathscr{F}}\right. & \left.\left(\mathscr{J}\left(\zeta^{*}\right), \zeta^{*}\right)\right) \leq f\left(d_{\mathscr{F}}\left(\mathscr{J}\left(\zeta^{*}\right), \mathscr{J}\left(\zeta_{n}\right)\right)+d_{\mathscr{F}}\left(\mathscr{J}\left(\zeta_{n}\right), \zeta^{*}\right)\right)+\tau \\
\leq & f\left(\varphi\left(\max \left\{d_{\mathscr{F}}\left(\zeta^{*}, \zeta_{n}\right), \frac{d_{\mathscr{F}}\left(\zeta^{*}, \mathscr{J}\left(\zeta^{*}\right)\right) d_{\mathscr{F}}\left(\zeta_{n}, \mathscr{J}\left(\zeta_{n}\right)\right)}{1+d_{\mathscr{F}}\left(\zeta^{*}, \zeta_{n}\right)}\right\}\right)\right. \\
& \left.+d_{\mathscr{F}}\left(\zeta_{n+1}, \zeta *\right)\right)+\tau, \\
< & f\left(\max \left\{d_{\mathscr{F}}\left(\zeta^{*}, \zeta_{n}\right), \frac{d_{\mathscr{F}}\left(\zeta^{*}, \mathscr{J}\left(\zeta^{*}\right)\right) d_{\mathscr{F}}\left(\zeta_{n}, \zeta_{n+1}\right)}{1+d_{\mathscr{F}}\left(\zeta^{*}, \zeta_{n}\right)}\right\}\right. \\
& \left.+d_{\mathscr{F}}\left(\zeta_{n+1}, \zeta *\right)\right)+\tau,
\end{aligned}
$$

for $n \in \mathbb{N}$. If $\max \left\{d_{\mathscr{F}}\left(\zeta^{*}, \zeta_{n}\right),\left(d_{\mathscr{F}}\left(\zeta^{*}, \mathscr{J}\left(\zeta^{*}\right)\right) d_{\mathscr{F}}\left(\zeta_{n}, \zeta_{n+1}\right) / 1\right.\right.$ $\left.\left.+d_{\mathscr{F}}\left(\zeta^{*}, \zeta_{n}\right)\right)\right\}=d_{\mathscr{F}}\left(\zeta^{*}, \zeta_{n}\right)$, then

$$
f\left(d_{\mathscr{F}}\left(\mathscr{J}\left(\zeta^{*}\right), \zeta^{*}\right)\right) \leq f\left(d_{\mathscr{F}}\left(\zeta^{*}, \zeta_{n}\right)+d_{\mathscr{F}}\left(\zeta_{n+1}, \zeta *\right)\right)+\tau,
$$

Letting $n \longrightarrow \infty$ and utilizing $\left(\mathscr{F}_{2}\right)$ and (35), we get

$$
\begin{aligned}
& \lim _{n \rightarrow \infty} f\left(d_{\mathscr{F}}\left(\mathscr{J}\left(\zeta^{*}\right), \zeta^{*}\right)\right) \\
& \quad \leq \lim _{n \rightarrow \infty} f\left(d_{\mathscr{F}}\left(\zeta^{*}, \zeta_{n}\right)+d_{\mathscr{F}}\left(\zeta_{n+1}, \zeta *\right)\right)+\tau=-\infty,
\end{aligned}
$$

which implies that $d_{\mathscr{F}}\left(\mathscr{J}\left(\zeta^{*}\right), \zeta^{*}\right)=0$, a contradiction.

If $\max \left\{d_{\mathscr{F}}\left(\zeta^{*}, \zeta_{n}\right),\left(d_{\mathscr{F}}\left(\zeta^{*}, \mathscr{J}\left(\zeta^{*}\right)\right) d_{\mathscr{F}}\left(\zeta_{n}, \zeta_{n+1}\right)\right) /(1+\right.$ $\left.\left.d_{\mathscr{F}}\left(\zeta^{*}, \zeta_{n}\right)\right)\right\}=\left(d_{\mathscr{F}}\left(\zeta^{*}, \mathscr{J}\left(\zeta^{*}\right)\right) d_{\mathscr{F}}\left(\zeta_{n}, \zeta_{n+1}\right)\right) /\left(1+d_{\mathscr{F}}\left(\zeta^{*}, \zeta_{n}\right)\right.$ ) , then

$f\left(d_{\mathscr{F}}\left(\mathscr{J}\left(\zeta^{*}\right), \zeta^{*}\right)\right) \leq f\left(\frac{d_{\mathscr{F}}\left(\zeta^{*}, \mathscr{J}\left(\zeta^{*}\right)\right) d_{\mathscr{F}}\left(\zeta_{n}, \zeta_{n+1}\right)}{1+d_{\mathscr{F}}\left(\zeta^{*}, \zeta_{n}\right)}+d_{\mathscr{F}}\left(\zeta_{n+1}, \zeta *\right)\right)+\tau$.

Letting $n \longrightarrow \infty$ and utilizing $\left(\mathscr{F}_{2}\right)$ and $(35)$, we get

$$
\begin{aligned}
& \lim _{n \rightarrow \infty} f\left(d_{\mathscr{F}}\left(\mathscr{J}\left(\zeta^{*}\right), \zeta^{*}\right)\right) \\
& \quad \leq \lim _{n \rightarrow \infty} f\left(\frac{d_{\mathscr{F}}\left(\zeta^{*}, \mathscr{J}\left(\zeta^{*}\right)\right) d_{\mathscr{F}}\left(\zeta_{n}, \zeta_{n+1}\right)}{1+d_{\mathscr{F}}\left(\zeta^{*}, \zeta_{n}\right)}+d_{\mathscr{F}}\left(\zeta_{n+1}, \zeta *\right)\right)+\tau=-\infty
\end{aligned}
$$

which implies that $d_{\mathscr{F}}\left(\mathscr{J}\left(\zeta^{*}\right), \zeta^{*}\right)=0$, a contradiction. Thus, we have $d_{\mathscr{F}}\left(\mathscr{J}\left(\zeta^{*}\right), \zeta^{*}\right)=0$, i.e., $\mathscr{J}\left(\zeta^{*}\right)=\zeta^{*}$.

For the uniqueness of the fixed point, we take the following property: of $\mathscr{J}$

(P) $\alpha(\zeta, \xi) \geq 1$ and $\beta(\zeta, \xi) \geq 1$ for all fixed points $\zeta, \xi \in \mathscr{M}$

Theorem 11. If we add the property $(P)$ in supposition of Theorem 10, then we get that the fixed point of the mapping $\mathscr{J}$ is unique.

Proof. Let $\zeta^{*}, \widehat{\zeta} \in \mathscr{M}$ be such that $\mathscr{J} \zeta^{*}=\zeta^{*}$ and $\mathscr{J} \widehat{\zeta}=\widehat{\zeta}$ such that $\zeta^{*}=\widehat{\zeta}$. Then, by hypothesis $(\mathrm{P})$, we have $\alpha\left(\zeta^{*}, \widehat{\zeta}\right) \geq 1$ and $\beta\left(\zeta^{*}, \widehat{\zeta}\right) \geq 1$. Suppose (i) holds. Then,

$$
\begin{aligned}
& d_{\mathscr{F}}\left(\zeta^{*}, \widehat{\zeta}\right)=d_{\mathscr{F}}\left(\mathscr{J}\left(\zeta^{*}\right), \mathscr{J}(\widehat{\zeta})\right) \\
& \leq \alpha\left(\zeta^{*}, \widehat{\zeta}\right) \beta\left(\zeta^{*}, \widehat{\zeta}\right) d_{\mathscr{F}}\left(\mathscr{J}\left(\zeta^{*}\right), \mathscr{J}(\widehat{\zeta})\right) \\
& \leq \varphi\left(\max \left\{d_{\mathscr{F}}\left(\zeta^{*}, \widehat{\zeta}\right), \frac{d_{\mathscr{F}}\left(\zeta^{*}, \mathscr{J}\left(\zeta^{*}\right)\right) d_{\mathscr{F}}(\widehat{\zeta}, \mathscr{J}(\widehat{\zeta}))}{1+d_{\mathscr{F}}\left(\zeta^{*}, \widehat{\zeta}\right)}\right\}\right) \\
& =\varphi\left(d_{\mathscr{F}}\left(\zeta^{*}, \widehat{\zeta}\right)\right)<d_{\mathscr{F}}\left(\zeta^{*}, \widehat{\zeta}\right),
\end{aligned}
$$

a contradiction. Hence, $\exists \zeta^{*} \in \mathscr{M}$ such that $\mathscr{J} \zeta^{*}=\zeta^{*}$. Suppose (ii) holds. Then, there exists $0<l \leq 1$ such that

$$
\begin{aligned}
& \left.\left(\alpha\left(\zeta^{*}, \widehat{\zeta}\right) \beta\left(\zeta^{*}, \widehat{\zeta}\right)+l\right)^{d_{\mathscr{F}}\left(\zeta^{*}, \widehat{\zeta}\right.}\right) \\
& \quad=\left(\alpha\left(\zeta^{*}, \widehat{\zeta}\right) \beta\left(\zeta^{*}, \widehat{\zeta}\right)+l\right)^{d_{\mathscr{F}}\left(\mathscr{F}\left(\zeta^{*}\right), \mathscr{F}(\widehat{\zeta})\right)} \\
& \quad \leq(1+l)^{\varphi\left(\mathscr{R}\left(\zeta^{*}, \widehat{\zeta}\right)\right)}
\end{aligned}
$$

which implies that

$$
\begin{aligned}
d_{\mathscr{F}}\left(\zeta^{*}, \widehat{\zeta}\right) \leq & \varphi\left(\max \left\{d_{\mathscr{F}}\left(\zeta^{*}, \widehat{\zeta}\right), \frac{d_{\mathscr{F}}\left(\zeta^{*}, \mathscr{J}\left(\zeta^{*}\right)\right) d_{\mathscr{F}}(\widehat{\zeta}, \mathscr{J}(\widehat{\zeta}))}{1+d_{\mathscr{F}}\left(\zeta^{*}, \widehat{\zeta}\right)}\right\}\right) \\
& =\varphi\left(d_{\mathscr{F}}\left(\zeta^{*}, \widehat{\zeta}\right)\right)<d_{\mathscr{F}}\left(\zeta^{*}, \widehat{\zeta}\right)
\end{aligned}
$$

a contradiction. Hence, $\exists \zeta^{*} \in \mathscr{M}$ such that $\mathscr{J} \zeta^{*}=\zeta^{*}$. Suppose (iii) holds. Then, there exists $l \geq 1$ such that

$$
\begin{aligned}
\left(d_{\mathscr{F}}\left(\zeta^{*}, \widehat{\zeta}\right)+l\right) & \leq\left(d_{\mathscr{F}}\left(\mathscr{J}\left(\zeta^{*}\right), \mathscr{J}(\widehat{\zeta})\right)+l\right)^{\alpha\left(\zeta^{*}, \widehat{\zeta}\right) \beta\left(\zeta^{*}, \widehat{\zeta}\right)} \\
& \leq \varphi\left(\mathscr{R}\left(\zeta^{*}, \widehat{\zeta}\right)\right)+l
\end{aligned}
$$

which implies that

$$
\begin{aligned}
d_{\mathscr{F}}\left(\zeta^{*}, \widehat{\zeta}\right) & \leq \varphi\left(\max \left\{d_{\mathscr{F}}\left(\zeta^{*}, \widehat{\zeta}\right), \frac{d_{\mathscr{F}}\left(\zeta^{*}, \mathscr{J}\left(\zeta^{*}\right)\right) d_{\mathscr{F}}(\widehat{\zeta}, \mathscr{J}(\widehat{\zeta}))}{1+d_{\mathscr{F}}\left(\zeta^{*}, \widehat{\zeta}\right)}\right\}\right) \\
& =\varphi\left(d_{\mathscr{F}}\left(\zeta^{*}, \widehat{\zeta}\right)\right)<d_{\mathscr{F}}\left(\zeta^{*}, \widehat{\zeta}\right),
\end{aligned}
$$

a contradiction. Hence, $\exists \zeta^{*} \in \mathscr{M}$ such that $\mathscr{J} \zeta^{*}=\zeta^{*}$.

Corollary 12. Let $\left(\mathscr{M}, d_{\mathscr{F}}\right)$ be an $\mathscr{F}$-metric space and $\mathscr{J}: \mathscr{M}$ $\longrightarrow \mathscr{M}$ be $\alpha$-admissible. Assume that these assertions hold:

(a) $\left(\mathscr{M}, d_{\mathscr{F}}\right)$ is $\mathscr{F}$-complete 
(b) $\exists \zeta_{0} \in \mathscr{M}$ such that $\alpha\left(\zeta_{0}, \mathscr{J}\left(\zeta_{0}\right)\right) \geq 1$

(c) $\mathcal{J}$ is continuous or if $\left\{\zeta_{n}\right\}$ is a sequence in $\mathscr{M}$ such that $\alpha\left(\zeta_{n}, \zeta_{n+1}\right) \geq 1$ for all $n$ and $\zeta_{n} \longrightarrow \zeta^{*} \in \mathscr{M}$ as $n$ $\longrightarrow \infty$, then $\alpha\left(\zeta_{n}, \zeta^{*}\right) \geq 1, \forall n \in \mathbb{N}$. If any one of these assertions hold:

$$
\alpha(\zeta, \xi) d_{\mathscr{F}}(\mathscr{J}(\zeta), \mathscr{J}(\xi)) \leq \varphi(\mathscr{R}(\zeta, \xi))
$$

(i) $\exists 0<l \leq 1$ such that

(1)

$$
(\alpha(\zeta, \xi)+l)^{d_{\mathscr{F}}(\mathscr{F}(\zeta), \mathscr{F}(\xi))} \leq(1+l)^{\varphi(\mathscr{R}(\zeta, \xi))}
$$

(ii) $\exists l \geq 1$ such that

(2)

$$
\left(d_{\mathscr{F}}(\mathscr{J}(\zeta), \mathscr{J}(\xi))+l\right)^{\alpha(\zeta, \xi)} \leq \varphi(\mathscr{R}(\zeta, \xi))+l
$$

where

$$
\mathscr{R}(\zeta, \xi)=\max \left\{d_{\mathscr{F}}(\zeta, \xi), \frac{d_{\mathscr{F}}(\zeta, \mathscr{J}(\zeta)) d_{\mathscr{F}}(\xi, \mathscr{J}(\xi))}{1+d_{\mathscr{F}}(\zeta, \xi)}\right\}
$$

$\forall \zeta, \xi \in \mathscr{M}$, then $\exists \zeta^{*} \in \mathscr{M}$ such that $\mathscr{J} \zeta^{*}=\zeta^{*}$.

Proof. Taking $\beta(\zeta, \xi)=1$ for all $\zeta, \xi \in \mathscr{M}$ in Theorem 10 .

Corollary 13. Let $\left(\mathscr{M}, d_{\mathscr{F}}\right)$ be an $\mathscr{F}$-metric space and $\mathscr{J}$ $: \mathscr{M} \longrightarrow \mathscr{M}$ be $\alpha$-admissible mapping such that

$$
\alpha(\zeta, \xi) d_{\mathscr{F}}(\mathscr{J}(\zeta), \mathscr{J}(\xi)) \leq \varphi(\mathscr{R}(\zeta, \xi))
$$

where

$$
\mathscr{R}(\zeta, \xi)=\max \left\{d_{\mathscr{F}}(\zeta, \xi), \frac{d_{\mathscr{F}}(\zeta, \mathscr{J}(\zeta)) d_{\mathscr{F}}(\xi, \mathscr{J}(\xi))}{1+d_{\mathscr{F}}(\zeta, \xi)}\right\},
$$

$\forall \zeta, \xi \in \mathscr{M}$. Suppose that the following assertions are satisfied:

(a) $\left(\mathscr{M}, d_{\mathscr{F}}\right)$ is $\mathscr{F}$-complete

(b) $\exists \zeta_{0} \in \mathscr{M}$ such that $\alpha\left(\zeta_{0}, \mathscr{J}\left(\zeta_{0}\right)\right) \geq 1$

(c) $\mathcal{J}$ is continuous or if $\left\{\zeta_{n}\right\}$ is a sequence in $\mathscr{M}$ such that $\alpha\left(\zeta_{n}, \zeta_{n+1}\right) \geq 1$ for all $n$ and $\zeta_{n} \longrightarrow \zeta^{*} \in \mathscr{M}$ as $n$ $\longrightarrow \infty$, then $\alpha\left(\zeta_{n}, \zeta^{*}\right) \geq 1, \forall n \in \mathbb{N}$. Then $\exists \zeta^{*} \in \mathscr{M}$ such that $\mathscr{J} \zeta^{*}=\zeta^{*}$

Proof. If only (i) holds in Corollary 12.

Corollary 14. Let $\left(\mathscr{M}, d_{\mathscr{F}}\right)$ be an $\mathscr{F}$-metric space and $\mathscr{J}$ $: \mathscr{M} \longrightarrow \mathscr{M}$ be $(\alpha, \beta)$-admissible mapping such that

$$
\alpha(\zeta, \xi) \beta(\zeta, \xi) d_{\mathscr{F}}(\mathscr{J}(\zeta), \mathscr{J}(\xi)) \leq \varphi\left(d_{\mathscr{F}}(\zeta, \xi)\right)
$$

$\forall \zeta, \xi \in \mathscr{M}$. Assume that these assertions hold:

(a) $\left(\mathscr{M}, d_{\mathscr{F}}\right)$ is $\mathscr{F}$-complete

(b) $\exists \zeta_{0} \in \mathscr{M}$ such that $\alpha\left(\zeta_{0}, \mathscr{J}\left(\zeta_{0}\right)\right) \geq 1$ and $\beta\left(\zeta_{0}, \mathscr{J}\left(\zeta_{0}\right)\right.$ ) 1

(c) $\mathscr{J}$ is continuous or if $\left\{\zeta_{n}\right\}$ is a sequence in $\mathscr{M}$ such that $\alpha\left(\zeta_{n}, \zeta_{n+1}\right) \geq 1$ and $\beta\left(\zeta_{n}, \zeta_{n+1}\right) \geq 1, \forall n$ and $\zeta_{n} \longrightarrow \zeta^{*}$ $\in \mathscr{M}$ as $n \longrightarrow \infty$, then $\alpha\left(\zeta_{n}, \zeta^{*}\right) \geq 1$ and $\beta\left(\zeta_{n}, \zeta^{*}\right) \geq$ $1, \forall n \in \mathbb{N}$. Then, $\exists \zeta^{*} \in \mathscr{M}$ such that $\mathscr{J} \zeta^{*}=\zeta^{*}$

Proof. If only hypothesis (i) holds in Theorem 10 and $\max \left\{d_{\mathscr{F}}(\zeta, \xi),\left(d_{\mathscr{F}}(\zeta, \mathscr{J}(\zeta)) d_{\mathscr{F}}(\xi, \mathscr{J}(\xi))\right) /\left(1+d_{\mathscr{F}}(\zeta, \xi)\right)\right\}=$ $d_{\mathscr{F}}(\zeta, \xi)$.

Following is Boyd and Wong type in the setting of $\mathscr{F}$ -metric space which is a consequence of Corollary 14.

Corollary 15. Let $\left(\mathscr{M}, d_{\mathscr{F}}\right)$ be an $\mathscr{F}$-metric space and $\mathscr{J}$ $: \mathscr{M} \longrightarrow \mathscr{M}$ be a self-mapping such that

$$
d_{\mathscr{F}}(\mathscr{J}(\zeta), \mathscr{J}(\xi)) \leq \varphi\left(d_{\mathscr{F}}(\zeta, \xi)\right)
$$

$\forall \zeta, \xi \in \mathscr{M}$. Assume that these assertions hold:

(a) $\left(\mathscr{M}, d_{\mathscr{F}}\right)$ is $\mathscr{F}$-complete

(b) $\mathscr{J}$ is continuous. Then, $\exists \zeta^{*} \in \mathscr{M}$ such that $\mathscr{J} \zeta^{*}=\zeta^{*}$

Proof. Taking $\alpha(\zeta, \xi)=\beta(\zeta, \xi)=1$ for all $\zeta, \xi \in \mathscr{M}$ in Corollary 14.

Example 16. Let $\mathscr{M}=\mathbb{R}$ and $\mathscr{F}$-metric $d_{\mathscr{F}}$ given by

$$
d_{\mathscr{F}}(\zeta, \xi)=\left\{\begin{array}{l}
2^{|\zeta-\xi|}, \quad \text { if } \zeta \neq \xi \\
0, \text { if } \zeta=\xi
\end{array}\right.
$$

Take $f(\varrho)=-1 / \varrho$ and $\tau=1$. Define $\mathscr{J}: \mathscr{M} \longrightarrow \mathscr{M}$ by

$$
\mathscr{J}(\zeta)= \begin{cases}2 \zeta, & \text { if } \zeta>1, \\ \frac{\zeta}{2}, & \text { if } 0 \leq \zeta \leq 1, \\ 0, & \text { otherwise. }\end{cases}
$$


Now, we define $\alpha, \beta: \mathscr{M} \times \mathscr{M} \longrightarrow[0,+\infty)$ by

$$
\alpha(\zeta, \xi)=\beta(\zeta, \xi)=\left\{\begin{array}{l}
1 \text { if } \zeta, \xi \in[0,1] \\
0, \text { if } \zeta, \xi \in[0,1]
\end{array}\right.
$$

Evidently, $\mathcal{J}$ is twisted $(\alpha, \beta)-\varphi$ rational contraction of type (i) with $\varphi(\iota)=k \iota, \forall \iota \geq 0$ and $k \in(0,1)$. Actually, $\forall \zeta, \xi \in$ $\mathscr{M}$, we have

$$
d_{\mathscr{F}}(\mathscr{F}(\zeta), \mathscr{J}(\xi)) \leq k\left(\max \left\{d_{\mathscr{F}}(\zeta, \xi), \frac{d_{\mathscr{F}}(\zeta, \mathscr{F}(\zeta)) d_{\mathscr{F}}(\xi, \mathscr{J}(\xi))}{1+d_{\mathscr{F}}(\zeta, \xi)}\right\}\right)
$$

All the conditions of Theorem 9 are satisfied. Hence, $\mathscr{J} 0=0$ which is unique.

\section{Applications in Dynamic Programming}

In this section, we now establish the solution of functional equations arising from dynamic programming related to multistage process $[11,12]$ as an application of Theorem 10. Recall that a dynamic programming problem is a decision-making problem in $n$ variables in which the problem being subdivided into $n$ subproblems (stages), each being a decision-making problem in one variable only. The decision is the "goodness" of a selected alternative depending on satisfying the optimal policy of the problem. The state of the system at any stage is regarded as the information that links the stages together, such that the optimal decisions for the remaining stages can be made. The state allows us to consider each stage separately and guarantees that the solution is feasible for all the stages. This setting formulates the problems of mathematical optimization and computer programming which are converted into the problems of functional equations

$$
\mu(\zeta)=\sup _{\xi \in D}\{f(\zeta, \xi)+K(\zeta, \xi, \mu(g(\zeta, \xi)))\}, \zeta \in W
$$

where $f: W \times D \longrightarrow \mathbb{R}$ and $K: W \times D \times \mathbb{R} \longrightarrow \mathbb{R}$ and $g$ : $W \times D \rightarrow W, W$ and $D$ are Banach spaces, precisely, $W$ is a state space and $D$ is a decision space.

Let $B(W)$ represent the set of all bounded real-valued functions defined on $W$. For $h \in B(W)$, consider

$$
\|h\|=\sup _{\zeta \in W}|h(\zeta)|
$$

Clearly, $(B(W),\|h\|)$ is a Banach space. We endow $B(W)$ with the $\mathscr{F}$-metric (with $f(\varrho)=\ln \varrho$ and $a=0$ ) defined by

$$
d_{\mathscr{F}}(h, k)=\sup _{\iota \in W}|h(\iota)-k(\iota)|
$$

Evidently, $\left(B(W), d_{\mathscr{F}}\right)$ is a $\mathscr{F}$-complete $\mathscr{F}$-metric space. To show the existence of a solution of (64), we define $\mathscr{J}: B$
$(W) \longrightarrow B(W)$ by

$$
\mathscr{J}(h)(\zeta)=\sup _{\xi \in D}\{f(\zeta, \xi)+K(\zeta, \xi, \zeta(g(\zeta, \xi)))\}
$$

$\forall h \in B(W)$ and $\zeta \in W$. Clearly, $\mathscr{J}$ is well-defined as $f$ and $K$ are bounded.

Theorem 17. Let $\mathscr{J}: B(W) \longrightarrow B(W)$ is given by (67) and assume that these assertions are satisfied: that

Assume that there exists $\Theta: B(W) \times B(W) \longrightarrow \mathbb{R}$ such

(i) $\Theta(h, k) \geq 0 \Rightarrow \Theta(\mathscr{J}(k), \mathscr{J}(h)) \geq 0$, where $h, k \in B($ $W)$

(ii) $|K(\zeta, \xi, h(\zeta))-K(\zeta, \xi, k(\zeta))| \leq \varphi(|h(\zeta)-k(\zeta)|)$, where $\varphi \in \Psi, h, k \in B(W), \Theta(h, k) \geq 0, \zeta \in W$ and $\xi$ $\in D$

(iii) if $\left\{h_{n}\right\}$ is a sequence in $B(W)$ such that $\Theta($ $\left.h_{n}, h_{n+1}\right) \geq 0$ for all $n \in \mathbb{N} \cup\{0\}$ and $h_{n} \longrightarrow h^{*}$ as $n \longrightarrow+\infty$, then $\Theta\left(h_{n}, h^{*}\right) \geq 0$ for all $n \in \mathbb{N}$ $\cup\{0\}$

(iv) there exists $h_{0} \in B(W)$ such that $\Theta\left(h_{0}, \mathcal{J}\left(h_{0}\right)\right) \geq 0$

Then, functional equation (64) has a unique and bounded solution.

Proof. Note that $\left(B(W), d_{\mathscr{F}}\right)$ is a $\mathscr{F}$-complete $\mathscr{F}$ - metric space. Let $\varepsilon$ be an arbitrary positive number and $h_{1}, h_{2} \in B($ $W)$ such that $\Theta\left(h_{1}, h_{2}\right) \geq 0$, then there exist $\xi_{1}, \xi_{2} \in D$ such that

$$
\begin{gathered}
\mathscr{J}\left(h_{1}\right)(\zeta)<f\left(\zeta, \xi_{1}\right)+K\left(\zeta, \xi_{1}, h_{1}\left(g\left(\zeta, \xi_{1}\right)\right)\right)+\varepsilon, \\
\mathscr{J}\left(h_{2}\right)(\zeta)<f\left(\zeta, \xi_{2}\right)+K\left(\zeta, \xi_{2}, h_{2}\left(g\left(\zeta, \xi_{2}\right)\right)\right)+\varepsilon, \\
\mathscr{J}\left(h_{2}\right)(\zeta) \geq f\left(\zeta, \xi_{2}\right)+K\left(\zeta, \xi_{2}, h_{2}\left(g\left(\zeta, \xi_{2}\right)\right)\right), \\
\mathscr{J}\left(h_{1}\right)(\zeta) \geq f\left(\zeta, \xi_{1}\right)+K\left(\zeta, \xi_{1}, h_{1}\left(g\left(\zeta, \xi_{1}\right)\right)\right) .
\end{gathered}
$$

Now, from (68) and (71), it follows easily that

$$
\begin{aligned}
\mathscr{J}\left(h_{1}\right)(\zeta)-\mathscr{J}\left(h_{2}\right)(\zeta) & <K\left(\zeta, \xi_{1}, h_{1}\left(g\left(\zeta, \xi_{1}\right)\right)\right)-K\left(\zeta, \xi_{1}, h_{2}\left(g\left(\zeta, \xi_{1}\right)\right)\right)+ \\
& \leq \mid K\left(\zeta, \xi_{1}, h_{1}\left(g\left(\zeta, \xi_{1}\right)\right)\right)-K\left(\left(\zeta, \xi_{1}, h_{2}\left(g\left(\zeta, \xi_{1}\right)\right)\right) \mid+\right. \\
& \leq \varphi\left(\left|h_{1}(\zeta)-h_{2}(\zeta)\right|\right)+\varepsilon .
\end{aligned}
$$

Hence, we get

$$
\mathscr{J}\left(h_{1}\right)(\zeta)-\mathscr{J}\left(h_{2}\right)(\zeta)<\varphi\left(h_{1}(\zeta)-h_{2}(\zeta)\right)+\varepsilon
$$

Similarly, from (69) and (70), we obtain

$$
\mathscr{J}\left(h_{2}\right)(\zeta)-\mathscr{J}\left(h_{1}\right)(\zeta)<\varphi\left(h_{1}(\zeta)-h_{2}(\zeta)\right)+\varepsilon .
$$


Therefore, from (73) and (74), we have

$$
\left|\mathscr{J}\left(h_{1}\right)(\zeta)-\mathscr{J}\left(h_{2}\right)(\zeta)\right|<\varphi\left(h_{1}(\zeta)-h_{2}(\zeta)\right)+\varepsilon
$$

that implies,

$$
d\left(\mathscr{J}\left(h_{1}\right), \mathscr{J}\left(h_{2}\right)\right) \leq \varphi\left(d\left(h_{1}, h_{2}\right)\right)+\varepsilon
$$

Since $\varepsilon>0$ is arbitrary, then

$$
d\left(\mathscr{J}\left(h_{1}\right), \mathscr{J}\left(h_{2}\right)\right) \leq \varphi\left(d\left(h_{1}, h_{2}\right)\right) .
$$

Define

$\alpha(h, k)=\beta(h, k)=\left\{\begin{array}{l}1 \text { if } \Theta(h, k) \geq 0, \text { where } h, k \in B(W), \\ 0, \text { otherwise. }\end{array}\right.$

Consequently, we have

$$
\alpha\left(h_{1}, h_{2}\right) \beta\left(h_{1}, h_{2}\right) d\left(\mathscr{J}\left(h_{1}\right), \mathscr{J}\left(h_{2}\right)\right) \leq \varphi\left(d\left(h_{1}, h_{2}\right)\right),
$$

that is, $\mathscr{J}$ is a twisted $(\alpha, \beta)-\varphi$-contractive mapping of type (i) with $\alpha(h, k)=\beta(h, k)=1$ for all $h, k \in B(W)$. Thus, by Theorem $10, \mathcal{J}$ has a fixed point. Thus, all the suppositions of Theorem 10 are satisfied. Therefore, there exists $h$, such that $\mathscr{J}(h)=h$, which is the bounded solution of the functional Equation (64).

\section{Conclusion}

In the present paper, we have defined the notions of twisted $(\alpha, \beta)$-admissible and twisted $(\alpha, \beta)-\varphi$-rational contractions in the context of $\mathscr{F}$-metric spaces and established some generalized fixed point results. As an application of our main results, we investigate the problem of dynamic programming related to multistage process which reduces to the problem of solving the functional equation.

\section{Data Availability}

No data were used to support this study.

\section{Conflicts of Interest}

The authors declare that they have no competing interests.

\section{Authors' Contributions}

All authors contributed equally and approved the final manuscript.

\section{References}

[1] M. Jleli and B. Samet, "On a new generalization of metric spaces," Journal of Fixed Point Theory and Applications, vol. 2018, article 128, 2018.

[2] A. Hussain and T. Kanwal, "Existence and uniqueness for a neutral differential problem with unbounded delay via fixed point results," Transactions of A. Razmadze Mathematical Institute, vol. 172, no. 3, pp. 481-490, 2018.

[3] L. A. Alnaser, J. Ahmad, D. Lateef, and H. A. Fouad, "New fixed point theorems with applications to non-linear neutral differential equations," Symmetry, vol. 11, no. 5, p. 602, 2019.

[4] L. A. Alnaser, D. Lateef, H. A. Fouad, and J. Ahmad, "Relation theoretic contraction results in F-metric spaces," Journal of Nonlinear Sciences and Applications, vol. 12, no. 5, pp. 337344, 2019.

[5] D. Lateef and J. Ahmad, "Dass and Gupta's Fixed point theorem in $\mathscr{F}$-metric spaces," Journal of Nonlinear Sciences \& Applications, vol. 12, pp. 405-411, 2019.

[6] A. E. Al-Mazrooei and J. Ahmad, "Fixed point theorems for rational contractions in $\mathscr{F}$-metric spaces," Journal of Mathematical Analysis, vol. 10, no. 6, pp. 79-86, 2019.

[7] A. Hussain, H. Alsulami, N. Hussain, and H. Farooq, "Newly fixed disc results using advanced contractions on $\mathscr{F}$-metric space," Journal of Applied Analysis \& Computation, vol. 10, no. 6, 2020.

[8] H. Işik, N. Hussain, and A. R. Khan, "Endpoint results for weakly contractive mappings in $\mathscr{F}$-metric spaces with application," International Journal of Nonlinear Analysis and Applications, vol. 11, pp. 351-361, 2020.

[9] S. A. Almezel, J. Ahmad, and G. Marino, "Fixed point theorems for generalized $(\alpha \beta-\psi)$-contractions in $\mathscr{F}$-metric spaces with applications,” Mathematics, vol. 8, p. 584, 2020.

[10] B. Samet, C. Vetro, and P. Vetro, "Fixed point theorems for $\alpha-\psi$-contractive type mappings," Nonlinear Analysis, vol. 75, no. 4, pp. 2154-2165, 2012.

[11] Z. D. Mitrović, H. Aydi, N. Hussain, and A. Mukheimer, "Reich, Jungck, and Berinde common fixed point results on $\mathscr{F}$-metric spaces and an application," Mathematics, vol. 7, p. 387, 2019.

[12] F. Vetro, “F-contractions of Hardy-Rogers type and application to multistage decision processes," Nonlinear Analysis: Modelling and Control, vol. 21, no. 4, pp. 531-546, 2016. 